\title{
WATTSBurning: Design and Evaluation of an Innovative Eco-Feedback System
}

\author{
Filipe Quintal, Lucas Pereira, Nuno Nunes, Valentina Nisi, and Mary Barreto \\ Madeira Interactive Technologies Institute, University of Madeira, Caminho da Penteada, \\ Funchal, 9020-105, Madeira, Portugal \\ \{filipe.quintal, lucas.pereira, mary.barreto\}@m-iti.org, \\ njn@uma.pt, valentina.nisi@gmail.com
}

\begin{abstract}
This paper reports a 15 weeks study of artistic eco-feedback deployed in six houses with an innovative sensing infrastructure and visualization strategy. The paper builds on previous work that showed a significant decrease in user awareness after a short period with a relapse in consumption. In this study we aimed to investigate if new forms of feedback could overcome this issue, maintaining the users awareness for longer periods of time. The study presented here aims at understanding if people are more aware of their energy consumption after the installation of a new, art inspired eco-feedback. The research question was then: does artistic eco-feedback provide an increased awareness over normal informative feedback? And does that awareness last longer? To answer this questions participants were interviewed and their consumption patterns analyzed. The main contribution of the paper is to advance our knowledge about the effectiveness of eco-feedback and provide guidelines for implementation of novel eco-feedback visualizations that overcome the relapse behavior pattern.
\end{abstract}

Keywords: Sustainability, Aesthetics, Eco-feedback, User Interfaces, Prototyping.

\section{Introduction}

Individual household consumption accounts for a significant part of the total worldwide energy consumption. For example domestic electricity is responsible for two thirds of the electricity used in the United States, 36\% of the greenhouse gasses, and $12 \%$ of the fresh water consumption [1]. In the European Union final energy consumption for households is about $31 \%$ of the total energy consumed, only second to transportation, which accounts for 36\%. Between 1985 and 1998, the actual amount of energy consumed per household remained nearly constant, but the growing number of households increased energy use by $4 \%$. This effect will have a dramatic global impact as developing countries contribute to the increase of households with energy access. Also while household electrical appliances are becoming more efficient, there are more of them and they are being used more often. Reports from the EU show that consumption by all-electrical appliances and lighting represents about $55 \%$ of the electricity used by households. These appliances include the six large consumers of 
electricity (refrigerators, freezers, washing machines, dish-washers, TVs and dryers), and many other small appliances. [2].

Studies have shown that individual activities can control up to $50 \%$ of residential electricity consumption, depending on the physical structure of the building and what set of appliances users can control [3]. Moreover, most people are in fact concerned about the consequences of their actions. However, they are also unaware of the impact of their daily activities and more importantly how they can change their behavior to reduce consumption. This gap between users concerns and their actual knowledge of energy consumption habits, motivated companies and researchers to develop technologies that present users with information about their consumption. This type of technology is commonly called eco-feedback and is defined as technology that provides feedback on individual or group behaviors with a goal of reducing environmental impact [4].

The work presented here is part of a broader sustainability interdisciplinary research project which involves the deployment of a combination of sensing and ecofeedback technologies to motivate and trigger people to think, act, reflect and consume sustainably.

\section{Related Work}

The advancement and availability of sensing systems for environmentally related activities (e.g., human activity inference [4]) and interactive displays to feedback this data (e.g., mobile phones) provides a rich space of prospects for new types of ecofeedback solutions [5]. Currently there is an increasing number of commercial applications that provide real time energy monitoring. These solutions range from low-cost single-outlet (Kill-a-Watt ${ }^{1}$ and Watts $\mathrm{Up}^{2}$ ) to medium cost whole house power consumption (CurrentCost ${ }^{3}, \mathrm{TED}^{4}, \mathrm{Efergy}^{5}, \mathrm{Owl}^{6}$, etc.) to higher cost ambient feedback solutions (Wattson ${ }^{7}$, Energy $\mathrm{Orb}^{8}$ ). Through these solutions, feedback is often presented as raw energy use (e.g., Watts), personal cost (e.g., money), or environmental impact (e.g., CO2 emissions). Furthermore, eco-feedback is also an increasingly important research arena, confirmed by the growing number of articles presented at top international venues like CHI, INTERACT, DIS and Ubicomp, which nowadays devote specific sessions to sustainability. As a consequence, literature is abundant in design strategies and guidelines to implement such systems [6, 7, 8, 9].

\footnotetext{
${ }^{1}$ http: / / www.p3international.com/products/special/p4400/ p4400-ce.html 
One of the main challenges of designing eco-feedback systems is to present to users, how individuals or group activities can impact the environment. Commercially available feedback systems, have tried to present this information as kilograms of $\mathrm{CO} 2$ emissions. Pierce et al. [10] surveyed several publications exploring the impact of eco-feedback technologies in energy consumption and on consumers' behaviors. The authors found out that the use of eco-feedback technology resulted on savings between five and twelve percent of daily energy consumption. They also concluded that when savings didn't happen, the eco-feedback was displayed too infrequently (monthly) and hence was disconnected from the consumption behavior. Egan [11] confirmed that receiving timely feedback is key to motivating behavior change. Moreover, Fischer reports that eco-feedback is more efficient when given frequently, clearly presented, using computerized tools and allowing historic or normative comparisons [12].

Normally eco-feedback is associated with behavior change. There is evidence that the single goal of saving money (less electricity you consume the less you pay), is not enough to motivate conspicuous behavior change - between 5 and $15 \%$ reduction on average [13]. Consolvo et al. [14] implemented a system that promotes a more physically active lifestyle. Here the authors propose eight qualities that a system should have in order to be well accepted by users: Abstract \& Reflective, Unobtrusive, Public, Aesthetic, Positive, Controllable, Trending / Historical and Comprehensive. These studies have encouraged research into non-traditional feedback systems, turning to digital art and disruptive design for help. In the digital art domain, efforts were made to raise awareness regarding their behaviors in relation to sustainability.

For example, digital artist Tiffany Holmes, visualizes energy consumption through an art installation situated in a public space [15]. Holmes uses digital art to display hidden data of real time usage of key resources (such as electric appliances) and providing an aesthetically striking visualization in the public space of the building hall. Dwellers were able to relate to the visualization as a community, negative feedback was avoided and people living in the building were empowered to act upon their consumption by getting to know data that wouldn't be available to them otherwise. Another example of digital art employed to raise awareness about energy consumption is the Helsinki based project presented in [16]. The project consists of a public installation, in which a green cloud proportional to the city energy consumption is laser projected onto the smoke generated by the chimney of a coal energy power plant in the city. During the seven days of the installation, the green projected cloud would grow and shrink in direct proportion to the city energy consumption. In the final day of the installation, residents were asked to unplug the devices to reduce consumption, in order to dramatically increase the size of the green cloud. This resulted in a reduction of the peak demand in $800 \mathrm{kVA}$ (approximately the same power generated by a windmill running for one hour).

Despite these developments the effectiveness eco-feedback is known to have problems. Peschiera, reports that that after a certain period of usage of the feedback devices, users consumption relapse to values prior to the study [17]. Holmes reported this phenomenon in [15] where it was possible to see that users gradually returning to their previous behaviors if feedback was less frequent or no longer present. Our own research [18] confirmed this: "We would check our consumption more often initially until we got a rough idea or perception of what our consumption was but after that it 
would less frequent ", furthermore we observed that this lack of attention starts to happen after the fourth week of usage.

\subsection{Previous Work}

Prior to the development and test of the WATTSBurning system, the research team was responsible for 2 long-term eco-feedback deployments. Those deployments involved 30 houses and apartments located in an urban area in southern Europe. To better understand our starting ground, we briefly discuss the results from those deployments that lead us to the implementation of the WATTSBurning system. A detailed explanation can be found in the corresponding reference.

\section{First Deployment.}

In the first deployment the research team designed an eco-feedback interface based on an evaluation of commercially available systems. The system presented real-rime and historical consumption using bar charts and involved three modes of operation: idle, attention and detail. These modes were triggered by a camera (using face and motion detection algorithms), which were also used to log user activity. The initial system was deployed in 21 houses for a period of 9 weeks. The data showed a $9 \%$ average aggregated decrease of consumption. A deeper look into the data disclosed that families that used the eco-feedback system more often had higher decreased consumption. Nevertheless there was a steep decrease in the users' interested in the eco-feedback, particularly after the first 4 weeks (users interest was measured by the amount of access to the different application features) [18].

\section{Second Deployment.}

Based on the findings of the first study, the feedback system and sensing infrastructure was completely redesigned and revised. The new eco-feedback interface was developed with the help of a designer following a set of "guidelines" extracted from the research literature. We conducted a 52 weeks long-term study using the revised system deployed in 13 houses starting from the first sample [19].

Results from the second study revealed that initially people interacted with the system more than with the previous one. However, the frequency of the interaction started to decrease after two weeks, and again the steepest decrease happened after four weeks confirming our initial findings [18]. Detailed analysis of interaction data revealed that after four weeks, some users even stopped checking the system altogether. A few others kept using it but less frequently (only once or twice a day). During this second deployment users' consumption remained virtually unchanged from the first deployment, suggesting that savings come from the initial understanding of consumption patterns. 


\section{Design Rationale}

The experience gained from the studies described above suggested that a different approach was required to overcome the decreased interest of users in energy data. Using inspiration from artist we brainstormed ideas and decided to change strategy and test a new paradigm of eco-feedback. We postulated a more inspiring and emotional visualization towards a less information driven eco-feedback system. After brainstorm sessions with artist designers and engineers it was decided to implement the feedback based on mapping the energy consumption of the household with elements of the local landscape, with the goal of leveraging the emotional connection of families with the local natural patrimonies, which is home of a UNESCO heritage forest. It is important to note that most of the inhabitants already feel a strong connection with the local forest. This was visible after the tragic mudslides in 2010 and the forest fires in 2011. A thorough description of this process can be found in [20].

\subsection{Pilot}

To test the concepts described above a novel interface was developed for the ecofeedback system. It consisted of a video based animation of a well-known forest site. Based on the consumption level several elements of that landscape would change. The electricity real time consumption was mapped as the movement of the clouds in the background, and by adding and removing animals in the landscape. More consumption meant that the clouds would pass by faster, and more appliances turned on or off meant more animals in the forest. This was a neutral feedback since more appliances being turned on or off does not necessary means more consumption, and the movement of the clouds does not have any direct negative meaning. Figure 1 shows these how these two feedback modes where displayed in the application.

This new eco-feedback version was tested in eight households during one month. Consumption and interaction data was saved and four of the eight families were interviewed. Quantitative data revealed no significant differences in the energy consumption of the families neither prior or during the study. However, families using this new version of the system had an increased number of interactions when compared with families that used a "traditional" eco-feedback device during the same period. Nevertheless there was still a decrease in the interaction along the period of the study, consistent with the response-relapse behavioral pattern reported above. However, this was not a linear decrease as there were several peaks in interaction during the month.

Qualitative analysis of the interviews revealed that neutral feedback was not well understood by users. Users that were exposed to previous versions of the feedback also missed out on the quantitative data provided previously:

“No I didn't relate it to my consumption levels. I wasn't even near to realize that (laughs). I was looking for data, logical data about it (...) I could see some extra elements, I thought you were decorating the landscape but I didn't understand why were they being placed there" Family 4 mother.

"This one is simpler, there's just the image and the consumption on the right. In the other one I could see the consumption in terms of the whole day and this was more elucidative. I find this one more interesting but we need some kind of heads ups about 
how can we see the consumption throughout the whole month, the whole week" Familiy 3 husband.

On the other hand after the artistically inspired mappings of the energy consumption to the landscape were explained to the users (during the final interview) they found this new feedback paradigm interesting, and suggested merging the landscape visualization with the quantitative data display:

"I think both are valid. Maybe there could be a symbiosis between the two. This one is more pleasant the other one is just data. What matters to me is to have the data, it's probably the most important for me. However, I like the way this one is presented, I feel it's more interactive than the other one" Family 3 husband.

Three of the four families interviewed suggested that the concrete data provided by the first two versions should be merged with the aesthetic pleasure of the natural landscape shared this view.

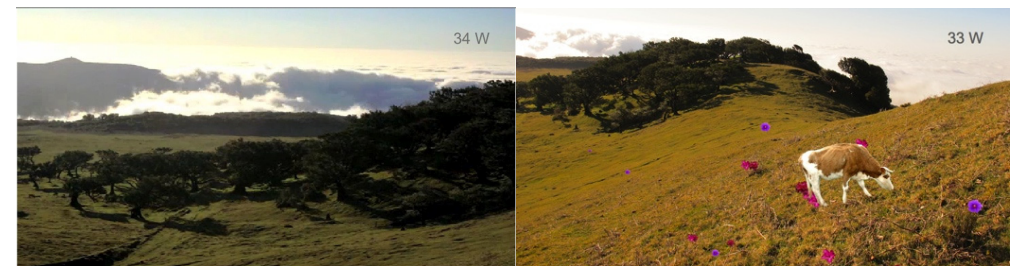

Fig. 1. Screenshot of the application used in the pilot. At left there's the main view of the forest, the consumption is mapped as the movement of the clouds in the background. At right there is the landscape with elements added based on the appliances used.

\subsection{Refinements of the Feedback System}

The experience from running the pilot of the WATTSBurning, made it clear that users found the feedback interesting, but they still wanted the concrete data about the consumption. Additionally the mapping between the forest and consumption was not fully understood and some users needed further explanation of how it was represented.

In the WATTSBurning system we also wanted to address some limitations found in the two previous deployments. In the initial deployments, the feedback was given via the display of a small netbook installed behind the main door of the house (where the main fuse box is located in most of the houses) [21], this type of stationary feedback is clearly limited since it is not accessible to all the household members at the time of decision (e.g. turning off a high consumption appliance). Also the fact the system was connected to the main fuse box made some families worry about the safety of the device, and sometimes would not allow children to use it. Furthermore some additional requirements emerge form the state of the art. The feedback should be accessible to all family members since the family dynamics and communication have an impact in the decision [22]. The system should allow the comparison between different periods (hour, day, weeks and months) so that people can explore and better understand their consumption patterns. The system should also provide simple tips about energy conservation and best practices promoting sustainable behavior change. The 
hardware itself should also be aesthetically pleasant, as Petersen [23], argues that pragmatist aesthetics is a promising approach for designing interactive systems as it promotes aesthetics of use, rather than aesthetics of appearance. Aesthetics play an increasingly important role in interaction design, in particular when designing for homes and everyday lives rather than for the workplace.

Collectively our prior experience and these requirements meant that the new version had to undergo major updates both on the software and in the hardware side. The eco-feedback device should ideally be portable so it can be accessed anywhere in the house (or outside). The sensing framework should provide data remotely to the eco-feedback device so that the sensing is removed from the house, addressing users safety concerns. The visual eco-feedback should be aesthetically pleasant while still providing concrete consumption information.

\subsection{System Design}

In order to provide accurate and meaningful eco-feedback, the system needs to measure the energy and resources consumption effectively. Measuring energy/resources consumption is in itself a challenging research problem. Researchers are striving to measure energy consumption in more cost-effective, accurate and less intrusive ways. One of the most promising research approaches is non-intrusive load monitoring (NILM), which reads data from a single point and tries to monitor and desegregate the consumption per appliance. The main assumption of most of the exiting NILM approaches is that every change in the total load consumption of a household happens as a response to an appliance changing its state. Therefore specific appliances can be isolated and their individual consumption calculated using complex signal processing and statistical learning techniques [18]. Low cost and non-intrusiveness are the main advantages of the NIML approach thus it was chosen as the building block of the sensing infrastructure that supports this research [21].

\subsection{Implementation}

In this section we explain how the requirements described in the sections above were implemented. Firstly we explain how the sensing framework evolved to the current version. Then we explain the implementation of the front end eco-feedback visualization of the system.

\section{Sensing Platform/Framework.}

Our sensing and eco-feedback platform is based on a custom made NILM system $[18,21]$. Our research was target at real world deployment of low-cost eco-feedback systems that are capable of sensing and disaggregating energy data in households. Our technical requirements were to find a low-cost solution that could run different NILM algorithms and eco-feedback visualizations while also collecting relevant user data. Originally our system was based on a netbook that acted as both as the sensing infrastructure (reading current and voltage from the microphone jack) and the visualization 
(via the built-in screen) and human sensing (via the built-in camera) system. Our initial prototype provided an easy way to deploy the system and evolve the visualizations during the first deployments. However, it was limited in terms of location (next to the mains at the entrance) and required installation inside the homes and unreliable Internet access. A second version of our system was implemented using a more capable DAQ (Data Acquisition Board) at the entrance of apartment buildings, hence measuring the energy consumption of multiple houses from one single location. This new version was less intrusive and enabled data to be pushed via web-services to different visualization platforms inside the home (web, tablets, etc.).

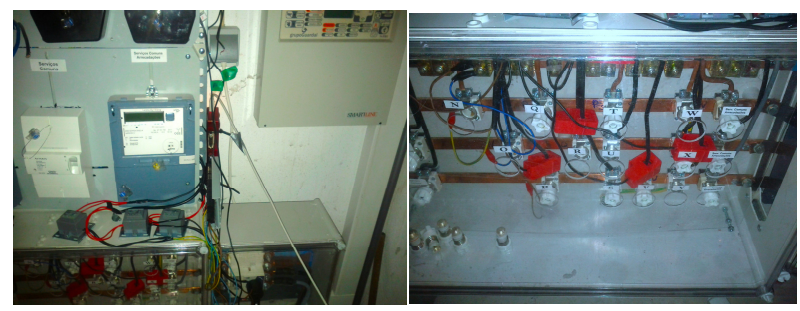

Fig. 2. Shows the system as it was deployed in one of the buildings of our study. At left there is the DAQ Board. At right there is the current clamp sensing each house.

Figure 2 shows the last implementation of our multi-apartment NILM sensing system. On the left hand-side one can see the three voltage sensors and the DAQ board, on the right it is possible to see current clamps installed on the current conductors of the apartments monitored. All of these signals are acquired and processed by a single computer that also runs the NILM algorithms and provides the data for eco-feedback devices via restful web services. The system also stores all the data in a database and provides access for consolidation in a datawarehouse.

\subsection{WATTSBurning Eco Feedback Visualization}

Following the requirements described before we implemented a new hardware and software platform but also modified the eco-feedback component, which now could be deployed on different portable devices. The new visualization addressed our experience in pilot (section 3.1) producing an aesthetically pleasant landscape that mapped the household consumption to elements of the natural forest. We also provided a way for users to access a second layer of more detailed consumption information.

The eco-feedback visualization was implemented on a 7', android tablet using the android native SDK (Figure 3). The tablet specifications allowed us to implement rich visualization, which can be accessed in different places of the house, or even outside. We believe that the device itself (a tablet) was still viewed as a novelty, and that helped our application to fit in the household as well as encouraging interaction.

The application receives real-time consumption and historical data from the sensing framework. The real time data is received using sockets and a custom made communication protocol. Historical data is gathered by using the web-services described in the previous sections. The historical data is also stored in the tablet so the 
users can still check their past consumption without an Internet connection. The transitions between the different modes of operation are stored locally enabling the analysis of the usage patterns by the research team. For example if a user picks up the tablet, presses the back button to go to the home screen and selects the daily consumption, the application will store 3 interactions with 3 different ids reffering to the 3 different views accessed by the user.

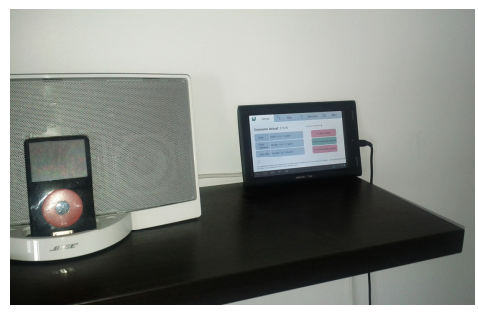

Fig. 3. System installed in one of the households

Our eco-feedback system involves two main modes of operation. When it is not used for two minutes it goes into the Energy Awareness mode that shows the consumption mapped as a digital illustration of the local endemic forest. Once the user interacts with the tablet, by pressing the back soft key, the system goes to Detailed Consumption mode and shows daily, weekly and monthly information about the home energy use. It is important to point that Energy Awareness was the default mode of the system, the tablet never went to an idle mode like it happens by default in some android applications.
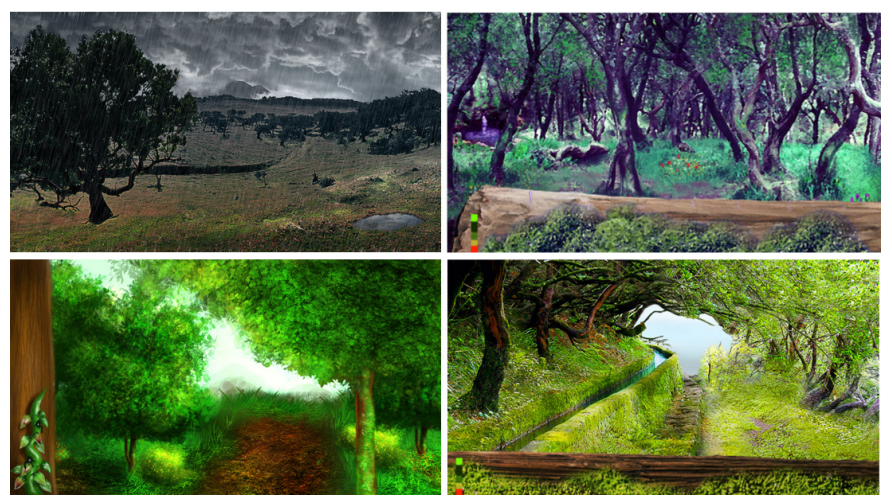

Fig. 4. Different landscape possibilities used during the think aloud process

\section{Energy Awareness Mode.}

To select the landscape and the metaphors used in this mode we performed a think aloud with two families (these families were later recruited as participants for this study). In these sessions the family members were presented with several paper prototypes displaying the consumption in different landscapes and represented with different items in the forest as seen in Figure 4. We decided to use a landscape of a 
well know forest site that was easily recognizable by the local community. The digitally modified pictures of the forest represented the comparison between the real time consumption and an average baseline consumption level. After the think aloud session we rejected some concepts like displaying the historical consumption as items in the landscape (e.g. moss growing on a tree) or the real time consumption mapped in the movment of an animals, these concepts were not clearly understood by the users.

In total there were five levels of consumption represented in the forest (as shown in Figure 5). These five levels represent when an household consumption is slightly above/bellow, well above/bellow or belongs to the average baseline. The baseline was composed of an average of the consumption on that period, for example the real time consumption on a Monday at 12:20 was compared against an average of all the Mondays during the period between 12:00 and 13:00. Aditional ilustrations of the forest are used to ensure a smooth transistion between the states, however the animation only stops in the five levels aforementioned.

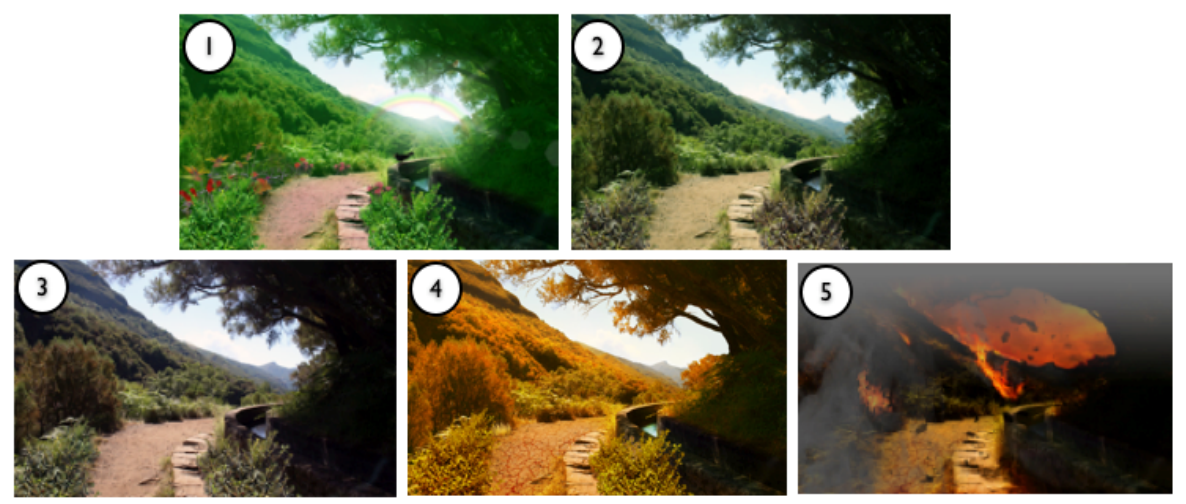

Fig. 5. Different views of the landscape according to the consumption. Ranging from low consumption (Image 1) to high consumption (image 5).

\section{Detailed Consumption Mode.}

This mode is triggered when the user presses the tablet back button. As a consequence the system presents a tabbed menu with four options: "Home", "Day", "Week" and "Month". The "Home" tab shows a summary of the overall consumption as well as the current real-time consumption (Figure 6 Left). The summary contains aggregated consumption of the current day/week and month, and comparisons between homologous periods. Also in this tab the user is presented with a "tip of the day" with general sustainable actions. The "Day" "Week" and "Month" tabs (Figure 6 Right) present a chart displaying the consumption over that period and the total aggregated consumption. It also informs the user of where the peak consumption happened and how it compares to the average of that period (for example in the "Week" tab the system shows how the consumption in the current week compares with an average of the previous weeks). By default the information presented here refers to the current day/week/month but the user can select preceding periods. 

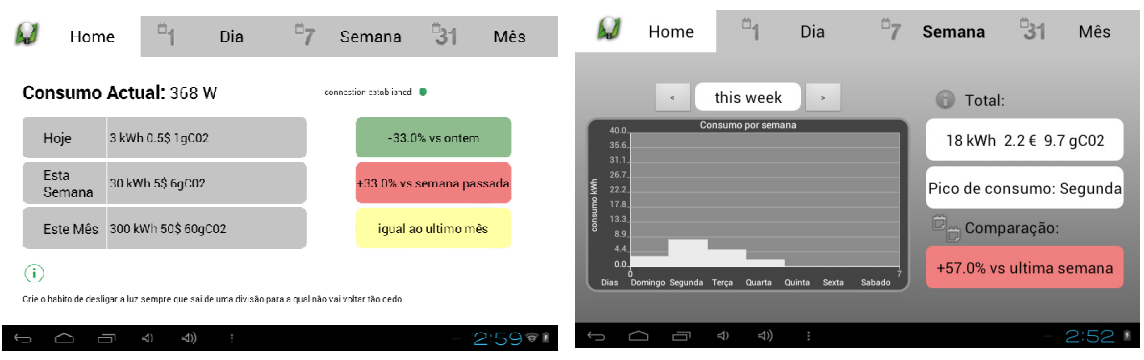

Fig. 6. Left: Home screen of the system. Right: Tab with the consumption of the current week

\subsection{Collected Data}

Our sensing framework samples the current and voltage waveforms with at a high sampling rate (about $3.2 \mathrm{kHz}$ ) but due to database size constraints only average power consumption is stored in a database (in 30 seconds sampling intervals). Additionally the application stores locally every transition between the different modes of operation of the eco-feedback system which is then periodically uploaded to one of our servers, hence allowing us to keep track of how the system is being used without having to wait for the end of the deployment.

The data analysed corresponded to two weeks of baseline consumption data collected when no eco-feedback was available and 15 weeks of consumption data after the eco-feedback was installed, as well as all the user interactions with the feedback during that time.

\section{Evaluation}

To test our system we recruited users from an apartment complex in an urban area in southern Europe. All the apartments were relatively new and they had similar appliances (provided by the construction company), consequently all of the families were relatively new to their houses. The sample is composed of six households: five couples with one or more children, and one young couple with no children. Two of the families had to leave the study earlier, but in both cases this was related to the fact that the families had to leave their apartments and not because they wanted to abandon the eco-feedback study. The families were told to use the tablet as they wanted (for example for browsing the web). But only the interactions in the WATTSBurning system were recorded, as described in the previous section.

\subsection{Method}

In order to assess the changes in consumption we collected baseline consumption data before the eco-feedback system was installed. This data was collected between the $13^{\text {th }}$ and the $27^{\text {th }}$ of August. After that each family received a tablet with the WATTSBurning application installed and given a short explanation of how to access the consumption data. After 22 days we visited the families and performed a short 
semi-structured interview. The families continued to use the system until the end of the year. Here we analyze the consumption and interaction data until the $17^{\text {th }}$ of December for a total of 17 weeks. We wanted to avoid the Christmas period, since the results in that period would most probably be biased.

\subsection{Qualitative Assessment}

When the families were interviewed after the first three weeks, we wanted to understand how the system was received, and if the Energy Awareness mode was being clearly understood and creating an increased awareness about energy consumption. It was also important to verify if any behavior change was triggered by the presence of the eco-feedback system. Five of the six families were interviewed, they will be referred as F1, F2, F3, F4, F5. We asked all the family members to be present in the interviews, in order to gather every member' opinion about the system. However, for F2 and F3 only the husbands were present during the interview.

The system was well received by all the families, and none of them had any major problems using it.

"I think it was simple, even the wireless connection was stable" F4

All the families agreed on the fact that the system increased their awareness about electricity consumption.

“It's raising my awareness, I don't think I've changed my consumption patterns yet, but I'm more aware now”. F1

“... if we have more devices turned on we can see right away there is an increase” F2

"It provides us with immediate feedback, such as daily and weekly consumption, and we can see what we do and how we behave in our daily routines to reduce our energy consumption" $\mathrm{F} 3$

"I got more alert, so necessarily I will try to consume less" F5

Another observation transversal to all of the families was that every family learned something about the consumption of certain devices.

"In the weekend for example, we were using the oven and I noticed it consumed a lot, then I turned on other devices out of curiosity" F1

"...the electric stove for example, I learned from the system that it consumed a lot" F2

"Especially the oven, it increases to 2000/2500W... I see there that it goes to $200{ }^{\circ} \mathrm{C}$ but I wasn't expecting so many watts... I was even surprised with the TV's” F3.

"I've learned that the oven consumes a lot, also the fridge" F5

It was also important to verify if the energy aware mode of the application was well understood. All families found that it was easy to relate their current consumption with the animation of the forest drying up and eventually being set on fire. It was also mentioned that the picture showing the state of the forest in the feedback interface worked as an alert of what was going on in the house, and it made them more aware of their consumption at specific moments. 
"Yes but.... For example when the dryer is working I see the forest catches fire, but I was expecting that, but I knew I couldn't keep that consumption for a long time, maybe I got more conscious” F1

"It gets dryer until it catches fires, when I have a lot of things turned on (...) also when there were only a few things on it showed rainbows and butterflies" F2

“(...) there's an association, the bigger the consumption the more destruction is visible in the forest, when we see it goes from green to yellow to red,... it's scary," F3.

All the families opted to place the system in a central place in the house, where it could be visible to all the family members. However in the five families that were interviewed it was mostly the adult male and young children who used it more often. "... I was curious in the beginning to see the forest on fire, but it was mostly my kid"F2

"It was mostly me and my son, my wife and my daughter didn't pay much attention to it, it was me and my younger son" $\mathrm{F} 3$,

"It was mostly me, she (girlfriend) wouldn't use the system".

Although the system was usually located in a central visible place in the house, it was common for participants to move it around, mostly because some of them wanted to check the consumption of a device in real time.

"It was mostly there, but it was in the kitchen for some time" F2

"It was there close to the sofa, because it had better reception for the internet (...) but I would also take it to the balcony while sitting there" $\mathrm{F} 3$

"I used it in different places in the house, I thought of it as one of those portable weather forecast displays that can be placed anywhere”. F5

\subsection{Quantitative Assessment}

From the qualitative assessment users reported a noticeable increase in their energy awareness. However, it is important to confirm if the reported awareness translates into an actual behavior change, confirmed by the quantitative data acquired from our sensing platform. In the following sections we analyze and discuss this issue.

\section{Energy Consumption}

In order to compare energy consumption we use the week as the standard period of time. This is the unit that best spans the routines of a family impacting their energy consumption. For instance, some families organize the major cleaning in a single day of the week, others the ironing. The week also comprises the working days and the weekend that usually correspond to very different routines. Therefore we compare the consumption data in the 15 weeks of eco-feedback deployment with the average of the two weeks of baseline data.

If we consider the 6 households as a whole, after 15 weeks the average weekly consumption dropped on average $2 \%$ from the initial baseline ( $\mathrm{n}=6, \mathrm{SD}=7.99 \%$ ). However, the standard deviation suggests that the savings were not constant across the houses. As a consequence we analyzed each house individually. Three families $(\mathrm{F} 1, \mathrm{~F} 2, \mathrm{~F} 5)$ reduced their consumption by $5 \%, 7 \%$ and $13 \%$ and their consumption 
was below the baseline for long periods - 12 weeks for F1, 11 weeks for F2 and 5 weeks for F5. The other three families consumed on average more than the baseline , respectively $10 \%(\mathrm{~F} 3), 1.5 \%(\mathrm{~F} 4)$ and $2.5 \%(\mathrm{~F} 6)$. These 3 houses were below the baseline average during 3, 6 and 6 weeks respectively. Figure 7 shows how each family's consumption changed during the study.
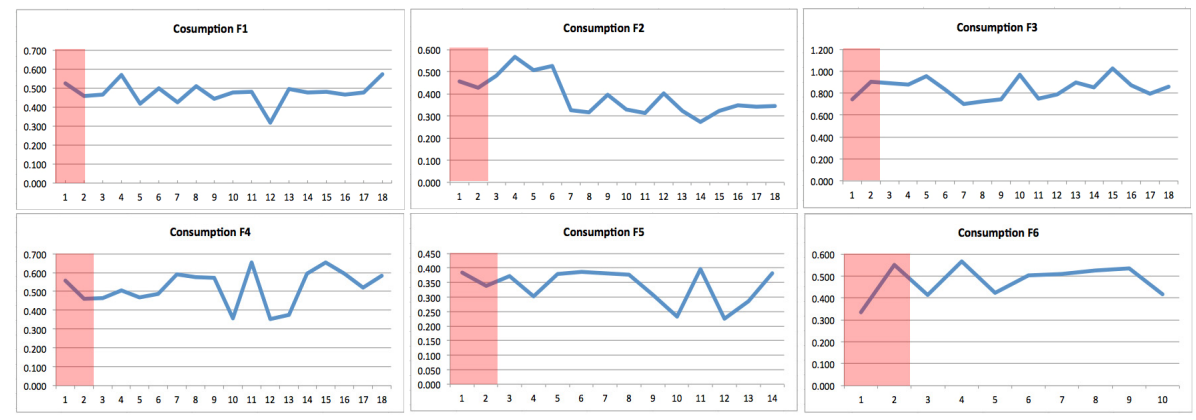

Fig. 7. Consumption in the 6 households during the study. The baseline period is highlighted in red. The horizontal axis represents the week in the study, and the vertical represents the average consumption in $\mathrm{kWh}$.

\section{Interactions with the System}

The analysis of the logged user interactions (the saved interactions are defined in section 3.6) showed that families used the system in completely different ways. Two of families (F3,F6) used the system more on the Mondays ,F1 and F4 used the system more on Wednesdays, F2 used the system mostly on Fridays and F1 on Tuesdays. This diversity was also noticeable when looking at how families used the system throughout the day. However, in al the families the view that was most triggered was the summary, followed by the animation view.

During the 15 weeks of the study a total of 1577 interactions with our system were logged among all of the households. That value represents an average of 266 interactions per house and an average of 16.3 interactions $(\mathrm{N}=1597, \mathrm{SD}=29)$ per day and 4.2 interactions per house and per day $(\mathrm{N}=6, \mathrm{SD}=6.06)$. Our system was mostly used in the afternoon (between 13:00 and 19:00), almost a third of the interactions happened during this period. It was also noticeable that all the families had a lot of interactions on the first four to five weeks of the study. After that the number of interactions dropped significantly (by more than two a thirds). Again the pattern was not similar in all houses, which justified the high standard deviation. Table 1 shows a summary of the number of interaction with the system, in the first four weeks of the study and rest of the period and in total. We choose to isolate the first four weeks because our previous research suggests that that after this period there is a steep drop in the usage of the feedback devices $[18,19]$.

To better understand the usage patters we analyze each household individually. The number of interactions on F1 only decreased by less than half, they kept using the system with an average of 14 interactions per day after the initial four-week period. Similarly with F2 the decrease in interaction was not considerable, but it was 
noticeable that they stopped using the system on a daily basis. Families 3, 4 and 5 had a lot of interactions in the first week, after that the interaction values dropped to less than one interaction per day ( $0.6,0.4$ and 0.2 per day respectively). F6 simply didn't interact directly with the system, even though this family agreed to participate in the study, they never showed much interest regarding the system nor they were ever available for interviewing.

We also analyzed the correlation of the user interactions with the energy consumption. The three households that on average didn't reduce their consumption are among the households that interacted less with the system (F4, F5 and F6). This finding is also consistent with our previous research.

Table 1. Summary of the average of interactions with the system by day, in different periods of the study

\begin{tabular}{llll}
\hline Family & \multicolumn{3}{c}{ Average interaction by day } \\
\cline { 2 - 4 } & First 4 weeks & Rest of the study & Total \\
F1 & 23.2 & 13.9 & 16.2 \\
F2 & 13.3 & 1.2 & 4.2 \\
F3 & 5.3 & 0.4 & 1.6 \\
F4 & 2.1 & 0.6 & 0.9 \\
F5 & 3.8 & 0.2 & 1.6 \\
F6 & 0.6 & 0 & 0.4 \\
\hline
\end{tabular}

It is important to note that the system only logged direct interaction with the interface. It would be very hard to count the number of time the users looked at the tablet with the Energy Aware mode on. Furthermore users confirmed that a significant amount of interactions were done with the tablet in the Energy Aware mode.

"It worked as an alert for me (...) it was easier to see from a distance (...) when we started to consume more it would get darker and catch fire” F5.

\section{Discussion}

The presence of eco-feedback increased users knowledge about the devices that they had at home. In fact all of the participants learned something about a particular appliance, this indicates a rise in the awareness about energy consumption, despite the level of usage or the change in overall consumption. However, the decreasing interest for eco-feedback after several weeks is an important factor leading to the relapse effect. Our novel eco-feedback system tried to overcome this issue by trying to map energy consumption to elements of the natural landscape. From the interviews with users we can conclude that the mapping the consumption with images of the local forest landscape was clear and well understood by participants. Although users didn't mention an emotional connection with the illustrated forest landscape, the energy awareness mode, where the forest dries up as more energy is consumed, did work as an alert for when consumption was higher than normal. Some users even found the forest on fire for a long time a disturbing factor that would motivate them to investigate which appliances were responsible for the higher level of consumption. Also, 
displaying the eco-feedback in a portable device allowed users to move the device through the household and explore the consumption of different appliances.

In terms of usage the system had a lot of interactions during the first four to five weeks, after that period the number of interactions decreased. However, unlike in our prior research the reduction was not linear and here were several peaks until the count finally settled at a low value. We believe this was a consequence of placement of the eco-feedback but also the presence of the energy awareness mode, which reminded people of the long-term consequences of their daily actions. The results in terms of user-interaction are an improvement over previous studies with classic quantitative forms of eco-feedback, since most families kept using the system after the four/five week period although less frequently and with different patterns. This difference in how families use the system is inline with the "one size does not fit all" [24] argument for eco-feedback systems. If we relate the quantitative interaction data with the consumption information it is noticeable that the houses that saved more energy are the ones that used the system more.

\section{Conclusions and Future Work}

In this paper we describe the studies and refinements leading to the design, prototyping and testing of WATTSBurning an eco-feedback device designed to foster awareness of energy consumption in households. Our research aimed at overcoming the know problem of people relapsing to previous behavior after several weeks of exposure to eco-feedback. Through an iterative design, testing and refinement process we improved our eco-feedback system introducing a new artistic metaphor that combines energy consumption levels to artistic representations of the local forest landscape. Our goal was to verify if this novel metaphor would improve on significant reduction of interaction with eco-feedback after four weeks of deployment. After initial prototyping that removed some ambiguity in the mappings of consumption to natural elements, the WATTSBurning system was successful in improving the levels of user attention and usage. Most families kept using the system even after four to five weeks, although to a lesser extent. The placement of the eco-feedback device and the presence of an energy awareness mode showing the landscape changing was an important motivator to retain user attention and awareness over time. These findings provide a good motivation to explore new forms of eco-feedback that go beyond traditional quantitative information. Clearly without more research eco-feedback technology will not confirm the promising results coming out of short-term three-week studies as the ones published in many HCI venues. The households in our study did manage to reduce their consumption, but they showed very different consumption and eco-feedback usage patterns. Overall households that used the system more saved more energy but there is still a lot to learn about the long-term consequences of ecofeedback. We believe our attempts with non-conventional forms of eco-feedback and, in particular, when exploring emotional and aesthetical aspects is a promising path to explore further.

Our deployment is still ongoing, and the plan is to further investigate the qualitative data. Relevant results might be achieved when comparing across houses or even 
between different periods in the same house. Furthermore our sensing framework is storing all the power events extracted from the NILM, which can also be useful to research into users consumption and behavior patterns. Even thought the feedback system was already removed the sensing framework is still gathering consumption data and it will be interesting to observe if there is any change in the consumption after the feedback is removed.

Acknowledgments. We would like to acknowledge designers Joana and Sofia Gomes for their contribution building the illustrations of the forest used the in WATTSBurning application. We would also like to thank all the families that took part of this study. The research presented here was funded by the SINAIS project (CMU - Portugal) CMU-PT/HuMach/0004/2008.

\section{References}

1. Wilson, A., Yost, P.: Buildings and the environment: the numbers. Environmental Building News 10 (2001)

2. European Environment Agency, http://www.eea.europa.eu/data-andmaps/figures/final-energy-consumption-by-sector-5 (retrieved on January 2013)

3. Schipper, L.: Linking lifestyle and energy used: a matter of time? Annual Review of Energy 14, 273-320 (1989)

4. Froehlich, J., Findlater, L., Landay, J.: The Design of Eco-Feedback Technology. In: CHI 2010, Atlanta. ACM (2010)

5. Mankoff, J., et al.: Leveraging Social Networks To Motivate Individuals to Reduce their Ecological Footprints. In: Proceedings of the 40th Annual Hawaii International Conference on System Sciences 2007, p. 87. IEEE Computer Society (2007)

6. Mills, E.: Review and comparison of web- and disk-based tools for residential energy analysis. Lawrence Berkeley National Laboratory, LBNL (2002)

7. Yolande, A.: Designing eco-feedback systems for everyday life. In: 2011 Annual Conference on Human Factors in Computing Systems, pp. 2135-2144. ACM (2011)

8. Mills, E.: Review and comparison of web- and disk-based tools for residential energy analysis. Lawrence Berkeley National Laboratory, LBNL (2002)

9. Consolvo, S., Everitt, K., Smith, I., Landay, J.A.: Design Requirements for Technologies that Encourage Physical Activity. In: CHI 2006, Montreal, pp. 457-466 (2006)

10. Pierce, J., Odom, W., Blevis, E.: Energy Aware Dweling: a critical survey for interaction design for eco-visualizations. In: OZCHI 2008. ACM Press, New York (2008)

11. Egan, C.: How customers interpret and use comparative displays of their home energy use. In: Proc. European Council for an Energy-Efficient Economy, Panel III, 19 (1999)

12. Dunlap, R.E.: Lay Perceptions of Global Risk. International Sociology 13(4), 473-498 (1998)

13. Fischer, C.: Feedback on household electricity consumption: a tool for saving energy? Energy Efficiency 1, 79-104 (2008)

14. Consolvo, S., McDonald, D.W., Landay, J.A.: Theory-driven design strategies for technologies that support behaviour change in everyday life. In: CHI 2009, New York, NY, USA, pp. 405-414 (2009) 
15. Holmes, T.G.: Eco-visualization: combining art and technology to reduce energy consumption. In: Proc. of the 6th ACM SIGCHI Conference on Creativity \& Cognition, pp. 153-162. ACM Press (2007)

16. Nuage Vert Nuage Vert Project (2008), http://www.pixelache.ac/nuageblog / (retrieved July 3, 2008)

17. Peschiera, G., Taylor, J.E., Siegel, J.A.: Response- relapse patterns of building ccupant electricity consumption following exposure to personal, contextualized and occupant peer network utilization data. Energy and Buildings 42, 1329-1336 (2010)

18. Nunes, N., Pereira, L., Quintal, F., Berges, M.: Deploying and evaluating the effectiveness of energy eco-feedback through a low-cost NILM solution. In: Proc. The 6th International Conference on Persuasive Technology (2010)

19. Pereira, L., Quintal, F., Barreto, M., Nunes, N.: Understanding the Limitations of Ecofeedback: A One Year Long-term Study. To appear at 1st SouthCHI (2013)

20. Nisi, V., Nicoletti, D., Nisi, R., Nunes, N.: Beyond eco-feedback: using art and emotional attachment to express energy consumption. In: Proceedings of C\&C, pp. 381-382. ACM (2011)

21. Pereira, L., Quintal, F., Nunes, N.J., Berges, M.: The Design of a Hardware-software Platform for Long-term Energy Eco-feedback Research. In: Proceedings of The ACM SIGCHI Symposium on Engineering Interactive Computing Systems (2012)

22. Barreto, M., Karapanos, E., Nunes, N.: Social Translucence as a theoretical framework for sustainable HCI. In: Campos, P., Graham, N., Jorge, J., Nunes, N., Palanque, P., Winckler, M. (eds.) INTERACT 2011, Part IV. LNCS, vol. 6949, pp. 195-203. Springer, Heidelberg (2011)

23. Petersen, M.G., et al.: Aesthetic interaction: a pragmatist's aesthetics of interactive systems. In: Proceedings of the 5th Conference on Designing Interactive Systems: Processes, Practices, Methods, and Techniques, pp. 269-276. ACM, Cambridge (2004)

24. He, H.A., Greenberg, S., Huang, M.E.: One size does not fit all: applying the Transtheoretical Model to Energy Feedback Technology Design. In: CHI 2010: Sense and Sustainability, pp. 927-936. ACM Press (2010) 